\title{
SOME RESULTS RELATED WITH BEREZIN SYMBOLS AND TOEPLITZ OPERATORS
}

\section{Mubariz T. Karaev, Mehmet Gürdal and Ulaş YamancI}

Abstract. We investigate some problems related with Berezin symbols of operators on Hardy and Bergman spaces and their applications in summability theory and in solution of Beurling problem. We also study boundedness and invertibility of some Toeplitz products on the Hardy and Bergman spaces.

Mathematics subject classification (2010): Primary 47B35.

Keywords and phrases: Reproducing kernel, Berezin symbol, Toeplitz operator, Hardy space, Bergman space.

\section{REFERENCES}

[1] N. AronZajn, Theory of reproducing kernels, Trans. Amer. Math. Soc. 68 (1950), 337-404.

[2] M. T. KARAEV, Berezin set and Berezin number of operators and their applications, The 8 th Workshop on Numerical ranges and numarical radii, WONRA 06, University of Bremen (Germany), July $15-17,2006$.

[3] M. T. KARAEV, Berezin symbol and invertibility of operators on the functional Hilbert spaces, J. Funct. Anal. 238 (2006), 181-192.

[4] M. Rosenblum and J. Rovnyak, Hardy classes and operator theory, Oxford Univ. Press, 1985.

[5] D. SARASON, Exposed points in $H^{1}, I I$, Operator theory: Adv. Appl. 41 (1990), 333-347.

[6] D. SARASON, Products of Toeplitz operators, in "Linear and Complex Analysis Problem Book 3", Part I, Lecture Notes in Mathematics, v. 1573, Springer-Verlag, New York, 1994.

[7] K. Stroethoff And D. Zheng, Invertible Toeplitz products, J. Funct. Anal. 195 (2002), 48-70.

[8] K. ZHU, Operator Theory in Function Spaces, Marcel Dekker, Ins. 1990. 